This is the seventh issue of Vital, and although now in only its third year of life it seems already to be very much a part of the BDA's stable of publications

\section{Pass it on}

Accompanying this issue you will find a copy of Vital, the $B D J$ supplement for the dental team. First and foremost - read it and pass it on to your dental team members it is for you and for them! The content is aimed specifically at the dental team, which as is often pointed out also includes dentists, and is intentionally designed to report on matters dental and non-dental that we believe are of interest and value to all in the team.

This is the seventh issue of Vital, and although now in only its third year of life it seems already to be very much a part of the BDA's stable of publications as we intend leading the way in which information on clinical dental practice will be shaped in the coming years.

In order to strengthen the links between publications, sharp eyed readers will note that one article in Vital is a digest of a paper previously published here in the $B D J$ about the education of hygienists in Scotland. In particular this looks at the challenges to be faced and overcome in relation to continuing professional development (CPD) for hygienists but touches on what is likely to become a problem for many in future, that of finding appropriate CPD and of funding it for all categories of PCD.

Also in Vital is a very enlightening feature on what being 'professional' means to the different groups comprising the dental team. The answers, given by a sample of team members taken from across the UK, are fascinating and I believe give an important insight into the ways in which dental care will be further elevated in the future. There is a general consensus that registration for the PCD groups is to be welcomed and supported. Being professional, the interviewees feel, is about being recognised as providing a significant and appreciated role in society but that, attendant upon the designation is also a duty to keep abreast of developments, to maintain standards and to be regarded as ethical. As dentists we are familiar with these requirements of being 'a professional' but it is exciting to read and

\section{OPINION}

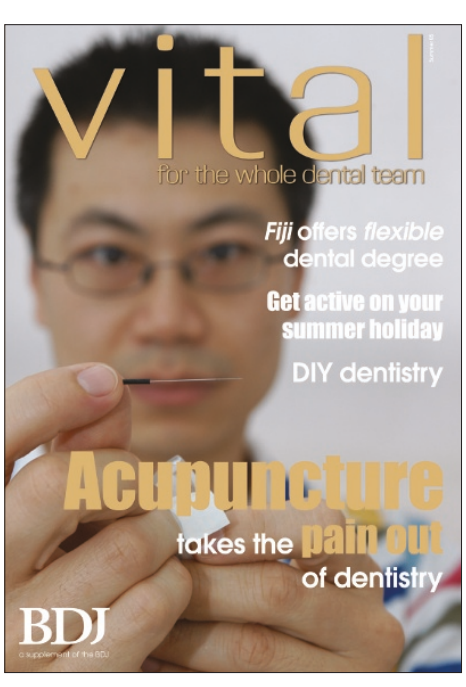

appreciate the enthusiasm of our colleagues as they anticipate accepting this mantle.

As importantly the 'Star letter' (page 4) is coincidentally penned by one of the co-authors of the 'Hygienists' paper, Margaret Ross, currently President of the British Dental Hygienists' Association. As you will read, in the letter Margaret points to the future role of the 'jointly' qualified dental

hygienist/therapist, who she speculates might be called an Oral Health Practitioner. Some readers will also have heard Margaret on a current UK roadshow describing more about the permitted duties of this new breed of colleague. For example, she points out that in addition to being able to undertake complex periodontal therapy, they are permitted to perform 'simple fillings'. Contrary to popular belief, this does not merely mean small occlusal cavities in deciduous teeth but, according to the definition in the regulations, it means any cavity in a child or adult tooth which does not involve the pulp. Take a moment to think about the significance of that.

Indeed, to add to the significance of that thought, at another presentation I was recently invited to attend, the speaker informed a somewhat stunned audience that, using the above definition over $70 \%$ of work currently paid for by the Dental Practice Board could currently and in future be undertaken by dental hygienist/therapists. While still very small in number compared with dentists, there can be little doubt that this presents an intriguing picture of possible models of future oral health care in the UK.

Whatever we might feel personally about these developments there is no doubt that oral care is going to have a very different shape in times to come, with a much trumpeted but now increasingly present role for additional dental team members. If you need further prompting, here it is - pass it on!

Stephen Hancocks OBE, Editor-in-Chief doi:10.1038/sj.bdj.4812451 